\title{
Annular Bragg defect mode resonators
}

\author{
Jacob Scheuer and Amnon Yariv \\ Department of Applied Physics, 128-95 California Institute of Technology, Pasadena, California 91125
}

Received April 10, 2003; revised manuscript received July 8, 2003; accepted July 11, 2003

\begin{abstract}
We propose and analyze a new type of a resonator in an annular geometry that is based on a single defect surrounded by radial Bragg reflectors on both sides. We show that the conditions for efficient mode confinement are different from those of the conventional Bragg waveguiding in a rectangular geometry. A simple and intuitive approach to the design of optimal radial Bragg reflectors is proposed and employed, yielding chirped gratings. Small bending radii and strong control over the resonator dispersion are possible by the Bragg confinement. A design compromise between large free-spectral-range requirements and fabrication tolerances is suggested. () 2003 Optical Society of America

OCIS codes: $130.0130,130.2790,230.5750$.
\end{abstract}

\section{INTRODUCTION}

The past few years have witnessed a substantial increase of activity in utilization of ring resonators for optical communication devices. Various ring-resonator-based applications such as modulators, ${ }^{1}$ channel drop filters, ${ }^{2}$ and dispersion compensators ${ }^{3}$ have been suggested and demonstrated.

The important characteristics of the modes of ring resonators are the free spectral range (FSR) and the loss per revolution, or, equivalently, the $\mathrm{Q}$ factor. One method of realizing tight confinement and high $\mathrm{Q}$ is to utilize Bragg reflection instead of total internal reflection (as in "conventional" resonators). Disk resonators based on Bragg reflection have been analyzed in the past, both for laser and passive-resonator applications, ${ }^{4-12}$ employing both coupled-mode theory and field transfer matrices.

In this paper we propose and analyze a new type of ring resonator: an annular-defect-mode resonator which consists of a single annular defect located between radial Bragg reflectors. Bragg-reflection-based disk resonators (i.e., a disk surrounded by concentric Bragg layers) and, recently, ring resonators have been studied theoretically and demonstrated experimentally. ${ }^{4-13}$ Also recently, a hexagonal-waveguide ring resonator based on photonicbandgap-crystal confinement on both sides of the waveguide was demonstrated experimentally. ${ }^{14}$ However, this structure exploited the specific symmetry of the triangular lattice that permits low-loss, $60^{\circ}$ abrupt turns in order to realize a closed resonator.

The basic geometry we propose is illustrated in Fig. 1. A circumferentially-guiding defect is located within a medium which consists of annular Bragg layers. As a result of the circular geometry, the layer widths, unlike in rectangular geometry, are not constant, ${ }^{15}$ and our task is to determine the widths that lead to maximum confinement in the defect.

In Section 2 we develop a matrix formalism in order to solve for the modal field, and in Section 3 we describe the rules for designing an annular Bragg-defect-mode resonator. In Section 4 we describe the dispersion relation and the modal profile of the field. In Section 5 we analyze the properties of resonators that are based on higher-Braggorder reflectors, and in Section 6 we discuss the results and summarize.

\section{BASIC THEORY}

We consider an azimuthally symmetric structure as illustrated in Fig. 1. The guiding defect, which is composed of a material with refractive index $n_{\text {def }}$, is surrounded by distributed Bragg reflectors on both sides, where the reflectors' layers are of refractive indices $n_{1}$ and $n_{2}$ alternating. All the electromagnetic field components can be expressed by the $z$ component of the electrical and magnetic field ${ }^{15}$ that satisfy the Helmholtz equation, which in cylindrical coordinates is given by

$$
\left[\frac{1}{\rho} \frac{\partial}{\partial \rho}\left(\rho \frac{\partial}{\partial \rho}\right)+\frac{1}{\rho^{2}} \frac{\partial^{2}}{\partial \theta^{2}}+k_{0}^{2} n^{2}(\rho)+\frac{\partial^{2}}{\partial z^{2}}\right]\left(\begin{array}{l}
E_{z} \\
H_{z}
\end{array}\right)=0,
$$

where $\rho, z$, and $\theta$ are the radial, axial, and azimuthal coordinates, respectively, and $k_{0}$ is the wave number in vacuum. The refractive index $n(\rho)$ equals $n_{\text {def }}, n_{1}$, or $n_{2}$ according to the radius $\rho$. Assuming that the $\rho, \theta$, and $z$ dependence of the field can be separated, the electrical field $z$ component of the electrical field can be written as

$$
E_{z}=R(\rho) \exp [i(m \theta \pm \beta z)] \quad m \text { is an integer, }
$$

with a similar expression for the magnetic field $z$ component. Introducing Eq. (2) into Eq. (1) leads to

$$
\rho^{2} \frac{\partial^{2} R}{\partial \rho^{2}}+\rho \frac{\partial R}{\partial \rho}+\left\{\left[k^{2}(\rho)-\beta^{2}\right] \rho^{2}-m^{2}\right\} R=0,
$$

where $k(\rho)=k_{0} n(\rho)$ is constant in each layer. The general solution of Eq. (3) can be expressed by a superposition of Bessel functions of the first and second kind:

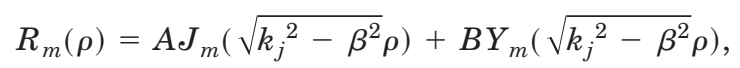

where $k_{j}$ is the material wave number in the $j$ th layer. By combining Eqs. (3) and (4), the electrical and magnetic $z$ components of the field become 


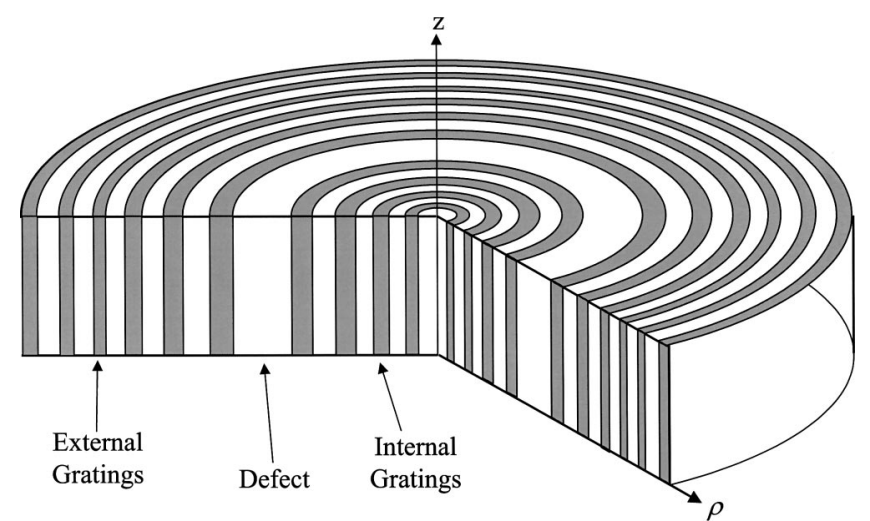

Fig. 1. Illustration of the annular-defect-mode resonator structure. Dark rings are of refractive index $n_{1}$, narrow light rings $n_{2}$, wide light ring $n_{\text {def }}$, center $n_{\text {core }}$.

$$
\begin{aligned}
E_{z}=[ & \left.A J_{m}\left(\sqrt{k_{j}^{2}-\beta^{2}} \rho\right)+B Y_{m}\left(\sqrt{k_{j}^{2}-\beta^{2}} \rho\right)\right] \\
& \times \cos (\beta z+\phi) \exp (i m \theta), \\
H_{z}= & {\left[C J_{m}\left(\sqrt{k_{j}^{2}-\beta^{2}} \rho\right)+D Y_{m}\left(\sqrt{k_{j}^{2}-\beta^{2}} \rho\right)\right] } \\
& \times \sin (\beta z+\phi) \exp (i m \theta) .
\end{aligned}
$$

The other fields' components are derived from $E_{z}$ and $H_{z}$ :

$$
\begin{aligned}
& E_{\theta}=\frac{i}{\gamma_{j}^{2}}\left(\omega \mu \frac{\partial H_{z}}{\partial \rho}-\frac{m}{\rho} \frac{\partial E_{z}}{\partial z}\right), \\
& E_{\rho}=\frac{1}{\gamma_{j}^{2}}\left(\frac{\partial^{2} E_{z}}{\partial z \partial \rho}-\frac{m \omega \mu}{\rho} H_{z}\right), \\
& H_{\theta}=\frac{-i}{\gamma_{j}^{2}}\left(\omega \epsilon \frac{\partial E_{z}}{\partial \rho}+\frac{m}{\rho} \frac{\partial H_{z}}{\partial z}\right), \\
& H_{\rho}=\frac{1}{\gamma_{j}^{2}}\left(\frac{\partial^{2} H_{z}}{\partial z \partial \rho}+\frac{m \omega \epsilon}{\rho} E_{z}\right),
\end{aligned}
$$

where $\gamma_{j}=\sqrt{{k_{j}{ }^{2}-\beta^{2}}^{2}}$, and $\epsilon$ and $\mu$ are the dielectric and magnetic susceptibilities, respectively.

Introducing Eq. (5) into Eq. (6) yields all the fields' components in the $j$ th layer. The parallel components of the fields- $E_{z}, H_{z}, E_{\theta}, H_{\theta}$-must be continuous at the interfaces. This requirement can be written in the form of a transfer matrix connecting the amplitude vector $[A B C D]$ in the $j$ th and $(j+1)$ th layers: where $\omega$ is the optical angular frequency, the prime indicates a derivative with respect to the function argument, and $\overline{\bar{M}}_{j}$ is the matrix to the right of the equal sign. The continuity consideration of the tangential electric and magnetic fields at the boundary $\rho_{j}$ separating the layers $j$ and $j+1$ leads to

$$
\left(\begin{array}{c}
A_{j+1} \\
B_{j+1} \\
C_{j+1} \\
D_{j+1}
\end{array}\right)=\overline{\bar{M}}_{j+1}{ }^{-1}\left(\rho_{j}\right) \cdot \overline{\bar{M}}_{j}\left(\rho_{j}\right) \cdot\left(\begin{array}{c}
A_{j} \\
B_{j} \\
C_{j} \\
D_{j}
\end{array}\right) .
$$

It is obvious from the structure of $\overline{\bar{M}}_{j}$ that the natural polarizations of the structure are not pure TE or TM. In this paper we are interested primarily in ring-resonator modes such that $\beta \approx 0$. In this case Eqs. (6) and (7) admit of two independent types of solutions: a TE mode with $E_{z}, H_{\rho}$, and $H_{\theta}$, and a TM mode with $H_{z}, E_{\rho}$, and $E_{\theta}$.

We consider the TE component of the electromagnetic field, which is characterized by $E_{z}, H_{\rho}$, and $H_{\theta}$. We designate this component as TE because the primary direction of the propagation is $\theta$. The $\overline{\bar{M}}$ matrix for this component is given by Eq. (7) with $\beta \approx 0$ :

$$
M_{j}=\left[\begin{array}{cc}
J\left(\gamma_{j} \rho\right) & Y\left(\gamma_{j} \rho\right) \\
\frac{n_{j}^{2}}{\gamma_{j}} J^{\prime}\left(\gamma_{j} \rho\right) & \frac{n_{j}{ }^{2}}{\gamma_{j}} Y^{\prime}\left(\gamma_{j} \rho\right)
\end{array}\right] .
$$

Using relation (8), the field components $A$ and $B$ can be "propagated" from the inner layers to the external layers. We use the finiteness of the field at $\rho=0$ so that $B_{1}$ $=0$. The second boundary condition is that beyond the last layer there is no inward-propagating field so that $B_{N+1}=-i A_{N+1}$ (for the TE mode), where $N$ is the number of layers.

The employment of the transfer matrices is important here because, in contrast to coupled mode theory, ${ }^{5,7}$ it permits an exact analysis of high-contrast Bragg structures that cannot be considered as small perturbations.

\section{DESIGN RULES}

The formalism of Section 2 enables us to find the modal field distribution in the case of an arbitrary arrangement of annular, concentric, dielectric rings. We are especially interested in structures that can lead to a concentration of the modal energy at a predetermined radial distance, i.e., in a radial defect.

High-efficiency Bragg reflectors in Cartesian coordinates require a constant grating period that determines

$$
\left(\begin{array}{c}
E_{z} \\
H_{\theta} \\
H_{z} \\
E_{\theta}
\end{array}\right)=\left[\begin{array}{cccc}
J\left(\gamma_{j} \rho\right) & Y\left(\gamma_{j} \rho\right) & 0 & 0 \\
\frac{n_{j}^{2}}{\gamma_{j}} J^{\prime}\left(\gamma_{j} \rho\right) & \frac{n_{j}^{2}}{\gamma_{j}} Y^{\prime}\left(\gamma_{j} \rho\right) & \frac{m \beta}{\rho \omega \epsilon_{0} \gamma_{j}^{2}} J\left(\gamma_{j} \rho\right) & \frac{m \beta}{\rho \omega \epsilon_{0} \gamma_{j}^{2}} Y\left(\gamma_{j} \rho\right) \\
0 & 0 & J\left(\gamma_{j} \rho\right) & Y\left(\gamma_{j} \rho\right) \\
\frac{m \beta}{\rho \omega \mu \gamma_{j}^{2}} J\left(\gamma_{j} \rho\right) & \frac{m \beta}{\rho \omega \mu \gamma_{j}^{2}} Y\left(\gamma_{j} \rho\right) & \frac{1}{\gamma_{j}} J^{\prime}\left(\gamma_{j} \rho\right) & \frac{1}{\gamma_{j}} Y^{\prime}\left(\gamma_{j} \rho\right)
\end{array}\right]\left(\begin{array}{c}
A_{j} \\
B_{j} \\
C_{j} \\
D_{j}
\end{array}\right] \equiv \overline{\bar{M}}_{j}(\rho)\left(\begin{array}{c}
A_{j} \\
B_{j} \\
C_{j} \\
D_{j}
\end{array}\right),
$$


the angles at which an incident wave would be reflected. Generally, the grating wave number $(2 \pi / \Lambda$, where $\Lambda$ is the grating period) multiplied by the reflection order should be approximately twice the transverse component of the incident wave's wave vector. ${ }^{15}$ However, when the structure is annular, the conditions for efficient reflection are different.

Several methods for determining the thickness, and thus the position, of the Bragg layers' interfaces have been suggested in previous publications. ${ }^{5-8}$ Compared with Bragg fibers, ${ }^{16}$ the incidence angle of the waves at the interfaces (measured from the normal to the interface) is smaller; therefore, the asymptotic approxima$\operatorname{tion}^{17}$ is not valid and the "conventional" $\lambda / 4$ layers would not be appropriate. The principle underlying these methods is to position the layers' interfaces at the zeros and extrema of the field transverse profile. This strategy ensures the decrease of the field intensity for larger radii and the reduction of radiating power from the resonator. Here we present a more intuitive, although equivalent, approach to determine the widths of the layers.

We use the conformal transformation ${ }^{18,19}$

$$
\begin{aligned}
& \rho=R \exp (U / R), \\
& \theta=V / R,
\end{aligned}
$$

and the inverse transformation

$$
\begin{aligned}
U & =R \ln (\rho / R), \\
V & =\theta R, \\
n(\rho) & =n_{\mathrm{eq}}(\rho) R / \rho,
\end{aligned}
$$

where $R$ is an arbitrary parameter. The transformation (10) maps a circle in the $(\rho, \theta)$ plane with radius $R_{0}$ to a straight line in the $(U, V)$ plane located at $U_{0}$ $=R \ln \left(R_{0} / R\right)$. The structure in Fig. 1 is transformed into a series of straight lines. The wave equation in the $(U, V)$ plane is obtained by transforming Eq. (1):

$$
\frac{\partial^{2} E}{\partial U^{2}}+\frac{\partial^{2} E}{\partial V^{2}}+k_{0}{ }^{2} n_{\mathrm{eq}}{ }^{2}(U) E=0,
$$

where $n_{\mathrm{eq}}(U)=n(U) \exp (U / R)$ is the profile of the refractive index in the $(U, V)$ plane. Figure 2(B) depicts the equivalent index profile $n_{\text {eq }}(U)$ in the $(U, V)$ plane corresponding to the real index profile $n(\rho)$ shown in Fig. 2(A). The latter exemplifies a "conventional" Bragg waveguide design comprising $\lambda / 4$ layers of alternating refractive index and a $\lambda / 2$ defect. As seen in Fig. 2(B), the equivalent index increases exponentially with the radius, and the equivalent grating period (which is constant in the real plane) also increases with the radius. This index profile does not necessarily support a guided-defect mode.

In the $(U, V)$ plane, the radial gratings are transformed into a series of parallel gratings normal to the $V$ axis but with an exponential index profile. For this structure to act as a Bragg reflector, the partial reflections from each interface must interfere constructively (see Fig. 3). For that to happen, the total phase shift that the wave accumulates while propagating through the layer should be $\pi / 2$. This condition determines the layer width as follows:

$$
\pi / 2=\int k_{\perp} \mathrm{d} U=\int \sqrt{k_{0}^{2} n_{\mathrm{eq}}{ }^{2}-\beta_{V}^{2}} \mathrm{~d} U,
$$

where the integration beginning and ending coordinates correspond to the interfaces of the layer, $n_{\text {eq }}=\bar{n} \rho / R$ and $\bar{n}=n_{j}{ }^{2} / \gamma_{j}$ according to Eq. (9). The propagation factor $\beta_{V}$ appearing in Eq. (13) is determined by the azimuthal wave number $m$ :

$$
\beta_{V}=m / R \text {. }
$$

Equation (13) was used to calculate the structure required for the high-reflection Bragg mirrors surrounding the defect. Assuming the Bragg reflectors on both sides have identical reflection phase, then the defect width must be $\lambda / 2$ in the sense of Eq. (13), i.e., the defect must satisfy

$l \pi=\int k_{\perp} \mathrm{d} U=\int \sqrt{k_{0}{ }^{2} n_{\mathrm{eq}}{ }^{2}-\beta_{V}^{2}} \mathrm{~d} U, \quad l=1,2,3 \ldots$,

where the integer $l$ indicates the number of the Bessel periods (or the radial modal number) of the field in the defect. It follows that the widths of the defect and the Bragg layers depend on their coordinate $U$ (or $\rho$ ) because the equivalent index $n_{\text {eq }}$ is a function of $U$.

(A)
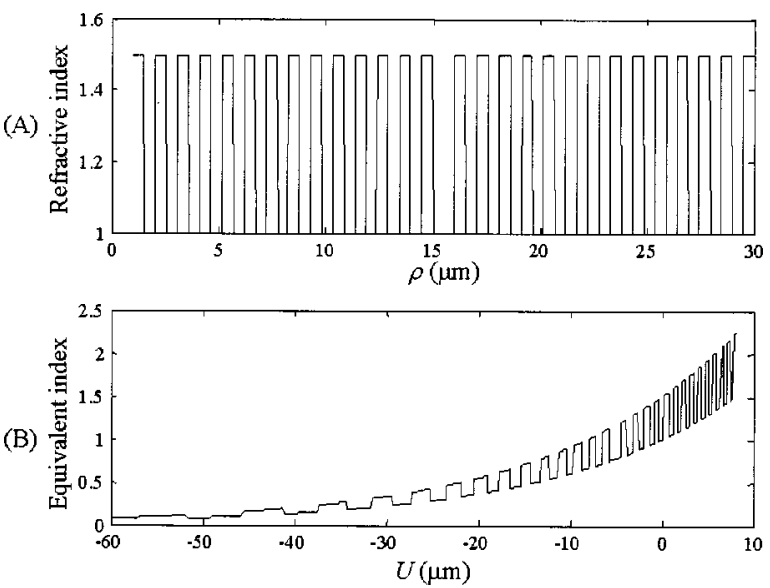

Fig. 2. Radial refractive index profile (A) and the equivalent index profile (B) of an annular defect surrounded by Bragg reflectors. The maximal and minimal refractive indices are 1.5 and 1 , respectively, and the grating period is $\sim 1 \mu \mathrm{m}$.

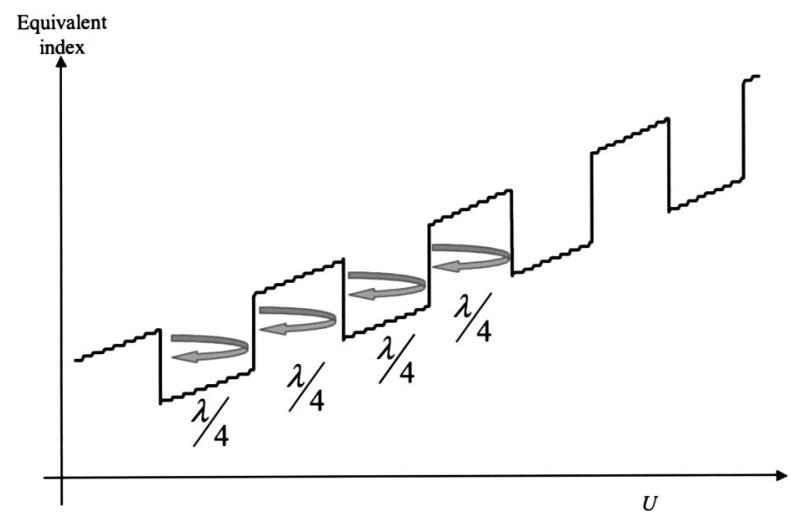

Fig. 3. Illustration of the design rule used to realize a highly efficient, radial Bragg reflector. 

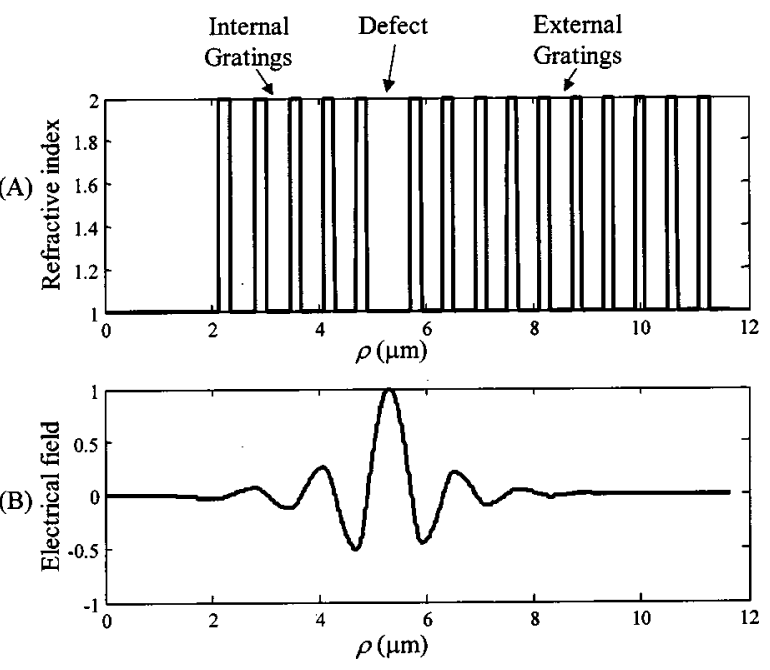

Fig. 4. Radial index profile (A) and electrical field distribution (B) of an annular-defect-mode resonator.

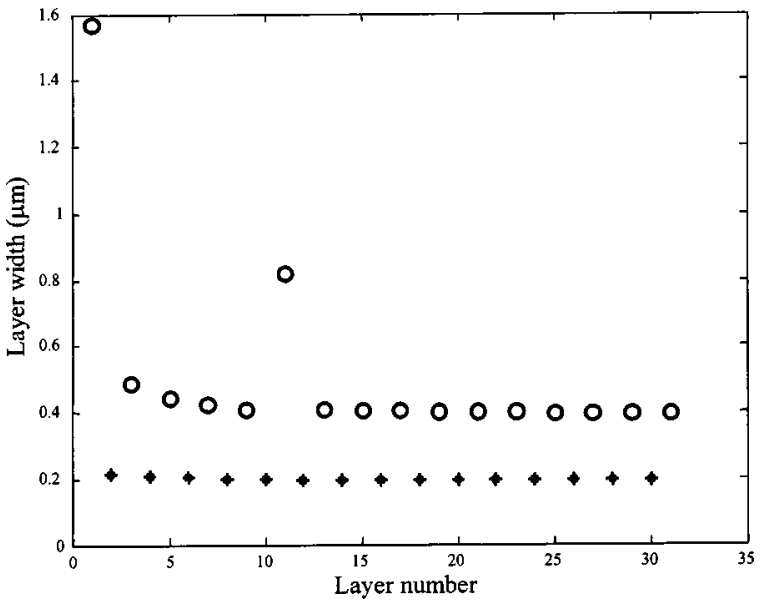

Fig. 5. High-index (stars) and low-index (circles) layer widths of the resonator shown in Fig. 4.

Figure 4 depicts the index (A) and the modal field (B) profiles of an annular defect mode resonator. The highindex layers have an effective refractive index $(\bar{n})$ of 2 while the low-index layers and the defect have an effective refractive index of 1 . The internal and external Bragg reflectors have 5 and 10 periods, respectively, the wavelength is $1.55 \mu \mathrm{m}$, and the azimuthal wave number is 7. The defect is located approximately at $\rho=5.6 \mu \mathrm{m}$ and it is $0.85 \mu \mathrm{m}$ wide.

Figure 5 shows the width of the high-index (stars) and low-index (circles) layers. At small radii the layers' width is greater because the equivalent index is lower there. The layers' width decreases for larger radii and approaches asymptotically the "conventional" quarterwavelength width $\lambda / 4 n$. The two exceptionally wide lowindex layers in Fig. 5 are the first low-index layer ( $\rho$ $=0-2 \mu \mathrm{m})$ and the defect, which has a $\lambda / 2$ width.

\section{MODAL SOLUTION PROPERTIES}

Because of the design method ( $\lambda / 4$ layers and $\lambda / 2$ defect), the resonator has a single radial mode whose peak is lo- cated almost exactly in the middle of the defect (see also Fig. 4). This is unlike the field profile of conventional ring resonators in which the field peak tends to shift toward the exterior radius of the waveguide as a result of the increase of the equivalent $n_{\text {eq }}$ index at larger radii. Nevertheless, the asymmetry of the field profile (with respect to the intensity peak) which is due to the radial structure is noticeable.

Figure 6 shows the dispersion curve of the annular defect resonator presented in Fig. 5. The vertical and horizontal axes indicate, respectively, the wavelength and the azimithal wave number $m$. The circles indicate the resonance wavelengths and the solid curve represents a quadratic interpolation: $\lambda=1.6309-4.8 \times 10^{-3}(\mathrm{~m})$ $-9.63 \times 10^{-5}\left(\mathrm{~m}^{2}\right)$. The resonator FSR at $\sim 1.55 \mu \mathrm{m}$ is approximately $20 \mathrm{~nm}$ and it increases for shorter wavelengths. It is interesting to note that the quadratic term is the most dominant term in the determination of the resonator FSR.

Figure 7 depicts the transverse profile of the modal fields corresponding to changing the azimuthal wave number from $m=6$ to 10 . It is evident that the trans-

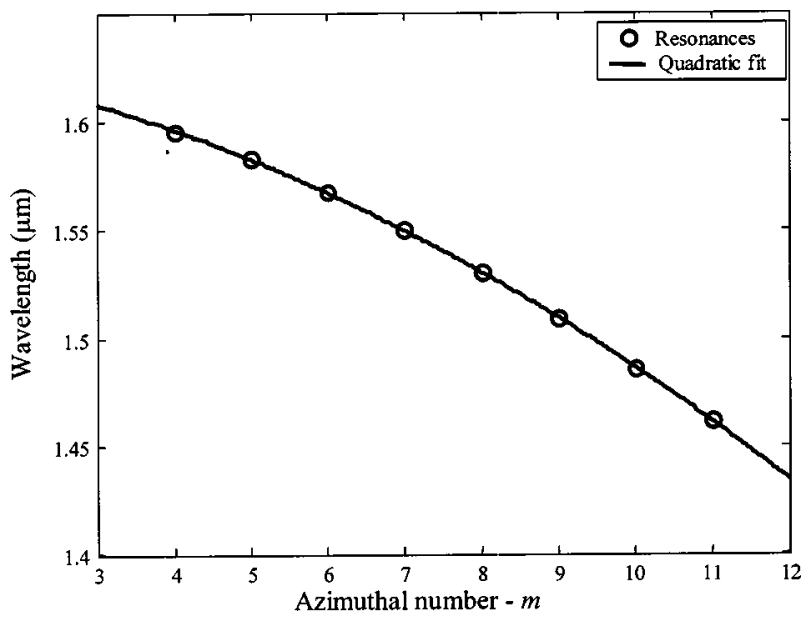

Fig. 6. Resonance wavelengths (circles) and quadratic fit (solid curve) of the resonator shown in Fig. 4.

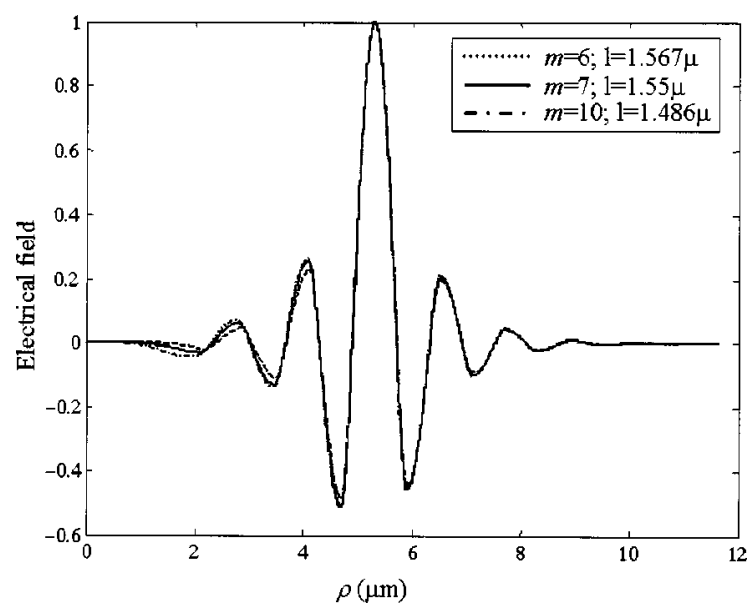

Fig. 7. Modal field profiles for $m=6$ (dotted curve), 7 (solid curve) and 10 (dashed-dotted curve) of the resonator shown in Fig. 4. 


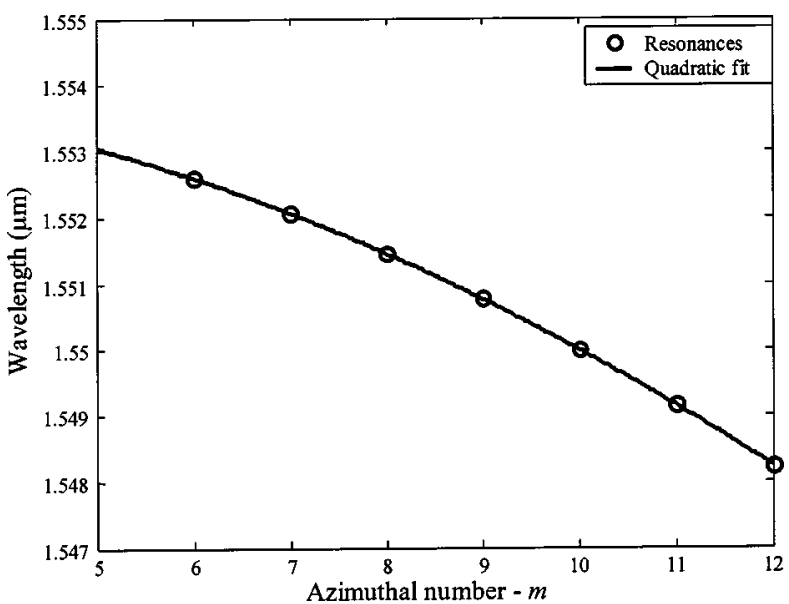

Fig. 8. Resonance wavelengths (circles) and quadratic fit (solid curve) of a resonator based on lower-contrast Bragg reflectors.

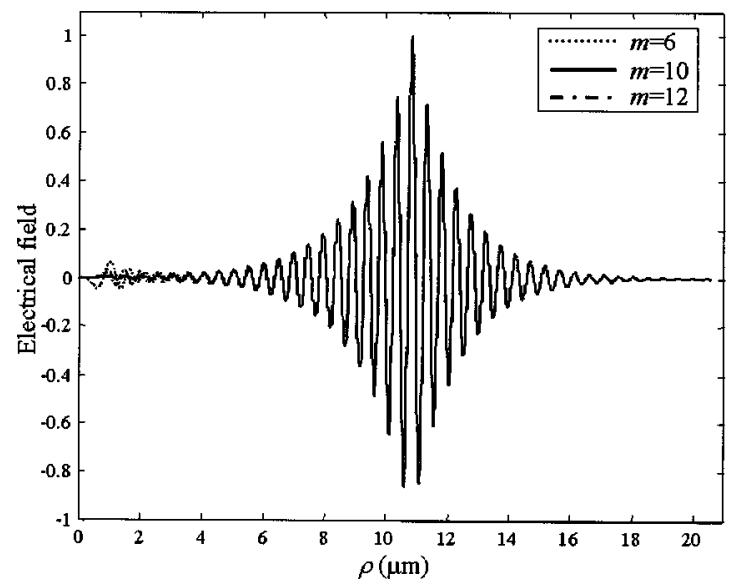

Fig. 9. Modal field profile of a resonator based on lower-contrast Bragg reflectors.

verse profile is almost identical although the resonance wavelength changes over more than $100 \mathrm{~nm}$. The reason for this is that the transverse profile is primarily determined by the Bragg layers width (or spatial frequency), which are independent of wavelength. This feature is an important advantage compared with conventional ring resonators because coupling between resonators of this type and Bragg waveguides, which is determined primarily by the modal profiles' overlap, can be expected to be almost wavelength independent. Figures 8 and 9 show the dispersion and the transverse profiles of $m=6$ to 12 for a Bragg defect resonator utilizing lower refractiveindex contrast. For this structure, $\bar{n}_{\text {def }}=\bar{n}_{2}=3.0$ and $\bar{n}_{1}=3.5$, the internal and external Bragg layers both have 40 periods, and they were designed for $m=10$ at $1.55 \mu \mathrm{m}$. The defect is located at $\rho=10.85 \mu \mathrm{m}$ and its width is approximately $0.27 \mu \mathrm{m}$. Because of the lower contrast, more Bragg layers are needed to realize good mode confinement, and as a result the resonator is larger and the FSR is smaller (about $96 \mathrm{GHz}$ at $1.55 \mu \mathrm{m}$ ).

As shown in Fig. 8, the dispersion curve of this resonator is also quadratic; it is given by $\lambda=1.5541-1.2$ $\times 10^{-5}(\mathrm{~m})-3.98 \times 10^{-5}\left(\mathrm{~m}^{2}\right)$. Similar to the high- contrast case, the modal transverse profile exhibits small wavelength dependence, which can be primarily seen in the small-radii regime.

\section{HIGHER-ORDER BRAGG REFLECTORS}

Although the chirped quarter-wavelength Bragg layers form an optimal reflector, their implementation could prove to be difficult. Because the layers' spatial period changes, some conventional photolithography methods that are employed for uniform (not chirped) Bragg gratings ${ }^{20}$ cannot be used. A possible approach to overcome this problem is to position the interfaces in nonsequential zeros-extrema, i.e., allow the Bessel function in each layer to complete a full period before changing the index. From the Bragg reflection point of view, such approach is equivalent to using $(2 l+1) \lambda / 4$ layers or employing higher reflection orders of the Bragg stack. Practically, the layer width can be evaluated in a manner similar to that of the quarter-wavelength structure, but the layers have to satisfy the following condition:

$$
\begin{aligned}
& (2 l+1) \pi / 2=\int k_{\perp} \mathrm{d} U=\int \sqrt{{k_{0}}^{2} n_{\mathrm{eq}}{ }^{2}-{\beta_{V}}^{2}} \mathrm{~d} U, \\
& l=1,2,3 \ldots .
\end{aligned}
$$

The resulting structure would have wider layers and would therefore be larger and exhibit smaller FSR. Figure 10 compares the field transverse profile of Fig. 4(A) and the transverse profile of a resonator designed for similar mode parameters ( 5 internal periods, 10 external periods, and $m=7$ for $\lambda=1.55 \mu \mathrm{m}$ ) but utilizing wider layers (B). The radius at which the field amplitude peaks is more than twice as large as the original (11.35 $\mu \mathrm{m}$ versus $5.6 \mu \mathrm{m}$ ), and the radial decay of the field rate is smaller. Figure 11 depicts the dispersion curve of the higher-Bragg-order-based resonator. As expected, the FSR of the resonator is significantly smaller than that of the original (approximately $3 \mathrm{~nm}$ at $1.55 \mu \mathrm{m}$ ). The main reason for this decrease in the FSR is the increase in the defect radius. However, since the low-index layers are inherently wider (especially in the lower-radii regime),

(A)
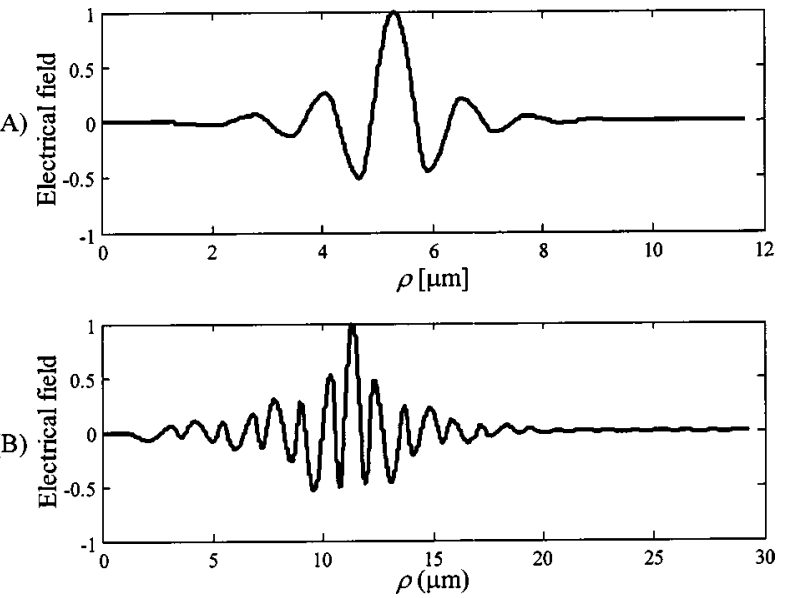

Fig. 10. Comparison of the modal field profile shown in Fig. 4(A) and the modal field of a resonator based on second-order Bragg reflectors with similar parameters $(\mathrm{B})$. 


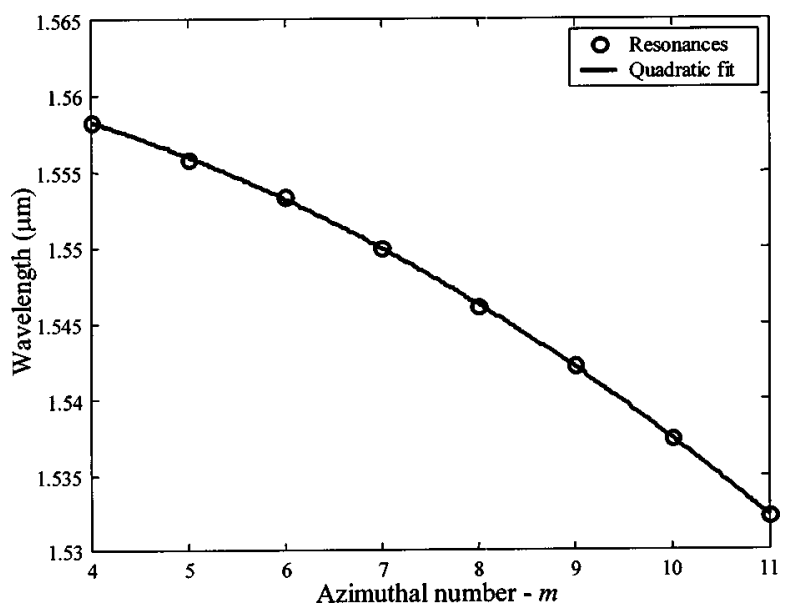

Fig. 11. Resonance wavelengths (circles) and quadratic fit (solid curve) of a second-order-Bragg-reflector-based resonator.

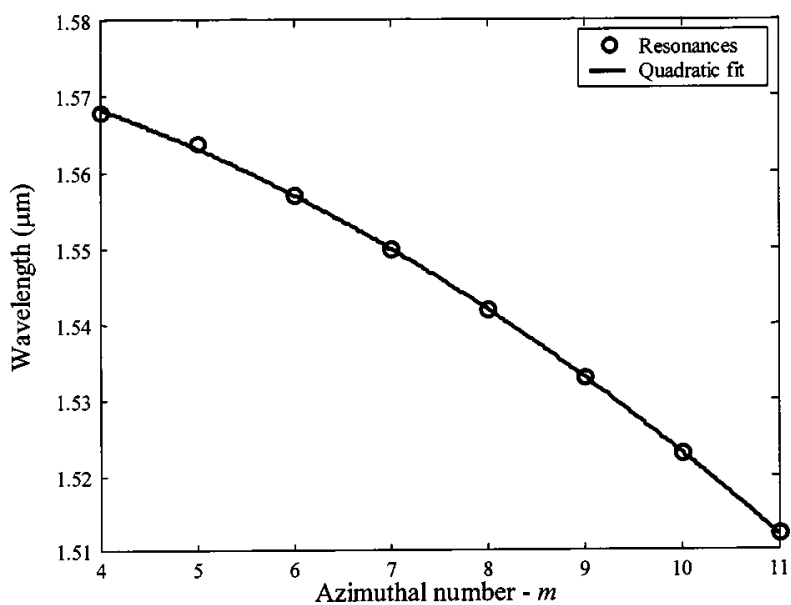

Fig. 12. Resonance wavelengths (circles) and quadratic fit (solid curve) of a resonator based on composite Bragg reflectors with parameters similar to those of the resonator of Fig. 4. The lowindex layers are quarter-wavelength in width and the high-index layers are three-quarter-wavelength in width.

they could be realized utilizing quarter-wavelength layers while the high-index layers would be realized as higherorder Bragg layers. Moreover, depending on the radius and the index, the Bragg order of each layer could be designed separately to achieve the largest FSR.

Figure 12 depicts the dispersion curve of a ring resonator designed for the same parameters as those of Figs. 4 and 9 in which the low-index layers are first-order Bragg layers and the high-index layers are second-order Bragg layers. The implementation of the composite structure increased the FSR from $3 \mathrm{~nm}$ to $\sim 8 \mathrm{~nm}$ without requiring smaller features. As with the other Bragg-defect resonators shown here, the modal transverse profile of this resonator is almost wavelength independent.

\section{DISCUSSION AND SUMMARY}

We have analyzed annular-defect-mode ring resonators based on radial Bragg reflectors. We have also presented a simple and intuitive method for designing the reflection gratings of such resonators. We have seen that ex- tremely small resonators (a few micrometers in diameter) exhibiting large FSR can be realized using relatively lowindex materials.

Several configurations to realize the Bragg reflectors have been suggested and analyzed. The straightforward configuration (each layer serves as an equivalent quarterwavelength plate) offers the smallest resonator exhibiting the largest FSR. On the other hand, manufacturing of such devices may require the realization of small and accurate features, especially if the optimal grating structure is required. Employment of higher-order Bragg gratings relaxes the tolerances on the gratings width but degrades FSR.

A composite configuration, i.e., tailoring each layer's Bragg order and width according to its refractive index and radius, seems to be the best compromise between large FSR and realizable features. Quarter-wavelength layers can be easily realized if the material refractive index is low or if the layer is positioned at a small radius where the equivalent index $n_{\text {eq }}$ is low. Employing the thinnest possible Bragg layers is important, especially for the internal Bragg reflector, because this would determine the defect radius, hence, the FSR. The external Bragg reflector could be realized by using higher-order Bragg layers without a major influence on the resonator performance. The more tolerant, higher-Bragg-order resonator design permits a relatively simple realization by conventional photolithography techniques in a variety of material systems such as GaAs, InP, and polymers.

\section{ACKNOWLEDGMENTS}

The authors thank Shayan Mookherjea and George T. Paloczi for useful discussions. This work was supported by the U.S. Office of Naval Research (Y. S. Park) and the U.S. Air Force Office of Scientific Research (H. Schlossberg).

Corresponding author J. Scheuer may be reached by e-mail to koby@caltech.edu.

\section{REFERENCES}

1. A. Yariv, "Critical coupling and its control in optical waveguide-ring resonator systems," IEEE Photon. Technol. Lett. 14, 483-485 (2002).

2. B. E. Little, "Ultracompact $\mathrm{Si}_{-} \mathrm{SiO}_{2}$ microring resonator optical dropping filter," Opt. Lett. 23, 1570-1572 (1998).

3. C. K. Madsen and J. H. Zhao, Optical Filter Design and Analysis: a Signal-Processing Approach (Wiley, New York, 1999).

4. M. Toda, "Single-mode behavior of a circular grating for potential disk-shaped DFB lasers," IEEE J. Quantum Electron. 26, 473-481 (1990).

5. X. H. Zheng and S. Lacroix, "Mode coupling in circularcylindrical system and its application to fingerprint resonators," J. Lightwave Technol. 8, 1509-1516 (1990).

6. T. Erdogan and D. G. Hall, "Circularly symmetric distributed feedback laser: an analysis," J. Appl. Phys. 68, 14351444 (1990).

7. T. Erdogan and D. G. Hall, "Circularly symmetric distributed feedback laser: coupled mode treatment of TE vector fields," IEEE J. Quantum Electron. 28, 612-623 (1992).

8. M. A. Kaliteevski, R. A. Abram, V. V. Nikolaev, and G. S. Sokolovski, "Bragg reflectors for cylindrical waves," J. Mod. Opt. 46, 875-890 (1999). 
9. C. Wu, M. Svilans, M. Fallahi, T. Makino, J. Glinski, C. Maritan, and C. Blaauw, "Optically pumped surfaceemitting DFB GaInAsP/InP lasers with circular grating," Electron. Lett. 27, 1819-1821 (1991).

10. D. Labilloy, H. Benisty, C. Weisbuch, T. F. Krauss, C. J. M. Smith, R. Hourdré, and U. Oesterle, "High-finesse disk microcavity based on a circular Bragg reflector," Appl. Phys. Lett. 73, 1314-1316 (1998).

11. A. Shaw, B. Roycroft, J. Hegarty, D. Labilloy, H. Benisty, C. Weisbuch, T. F. Krauss, C. J. M. Smith, R. Stanely, R. Hourdré, and U. Oesterle, "Lasing properties of disk microcavity based on circular Bragg reflector," Appl. Phys. Lett. 75, 3051-3053 (1999).

12. D. Ochoa, R. Hourdré, M. Ilegems, H. Benisty, T. F. Krauss, and C. J. M. Smith, "Diffraction of cylindrical Bragg reflectors surrounding an in-place semiconductor microcavity," Phys. Rev. B 61, 4806-4812 (2000).

13. J. Scheuer and A. Yariv, "Two-dimensional optical ring resonators based on radial Bragg resonance," Opt. Lett. 28, 1528-1530 (2003).

14. S. Kim, H. Ryu, H. Park, G. Kim, Y. Choi, Y. Lee, and J.
Kim, "Two-dimensional photonic crystal hexagonal waveguide ring laser," Appl. Phys. Lett. 81, 2499-2501 (2002).

15. See for example, A. Yariv, Optical Electronics in Modern Communications, 5th ed. (Oxford University, New York, 1997).

16. P. Yeh, A. Yariv, and E. Marom, "Theory of Bragg fiber," J. Opt. Soc. Am. 68, 1196-1201 (1978).

17. S. G. Johnson, M. Ibanescu, M. Skorobogatiy, O. Weisberg, T. D. Engeness, M. Soljačić, S. A. Jacobs, J. D. Joannopoulos, and Y. Fink, "Low-loss asymptotically single-mode propagation in large-core OmniGuide fibers," Opt. Express 9, 748-779 (2001).

18. M. Heiblum and J. H. Harris, "Analysis of curved optical waveguides by conformal transformation," IEEE J. Quantum Electron. QE-11, 75-83 (1975).

19. L. Djaloshinski and M. Orenstein, "Disk and ring microcavity lasers and their concentric coupling," IEEE J. Quantum Electron. 35, 737-744 (1999).

20. G. Meltz, W. W. Morey, and W. H. Glenn, "Formation of Bragg gratings in optical fibers by a transverse holographic method," Opt. Lett. 14, 823-825 (1989). 